\title{
Disease Identification using Retinal Vessel Segmentation using MATLAB
}

\author{
P Ramani, Radhika Baskar, Ajay Swaminathan G
}

\begin{abstract}
The state of the vascular system of the human eye is an imperative demonstrative factor in ophthalmology. The Project proposes the Retinal picture investigation through the productive discovery of vessels and exudates for retinal vasculature issue examination. It accepts basic occupations in acknowledgement of specific diseases in starting occasions, for instance, diabetes, which is performed by examination of the states of retinal veins.The natural attributes of retinal pictures make the vein location process troublesome. Here, we proposed another calculation to recognize the retinal veins more accurately.
\end{abstract}

Keywords: vascular system, ophthalmology, diabetes, MATLAB.

\section{INTRODUCTION}

Retinal vessel division is used in screening software for diabetic retinopathy, analysis of the retinopathy of indiscretion, affirmation of macular a vascular district, the region of arteriolar narrowing, estimation of vessel tortuosity to portray hypertensive retinopathy [1], estimating vessel broadness to stall high blood pressure, cardiovascular infirmities, and PC helped laser remedial framework. Other aberrant applications join the changed time of pupil of trademark inspirations driving the visual cortex vasculature for transient or multimodal picture determination, retina [2-3] picture mosaic blend, prominent affirmation of the optic plate location, and control of the fovea. Also, the game plan of retinal vessels is sufficiently irrefutable to every person and can be utilized for somatic perceiving confirmation notwithstanding the way that it has not been comprehensively investigated [4].

Fovea, optical circle and vessels are three most essential configuration of human retina and commonly utilized for a few uses. Exposure of these fundamental structures physically is dull [5]. It relies on the strength of client. The two most persuading segments that irregular establishment lighting up is from the verifying system and the way that assorted vessels have particular show up diversely in connection to the establishment [6-7]. Likewise, we propose a modified vein conspicuous confirmation count that segments veins of the retina with high precision in a base

Revised Manuscript Received on December 11, 2019

* Correspondence Author

P Ramani *, SRM Institute of Science and Technology, Chennai, India Email: ramanivijisachin79@gmail.com

Radhika Baskar, Saveetha School of Engineering, SIMATS, Chennai, India. Email: radhikabaskar@saveetha.com

Ajay Swaminathan G, Saveetha School of Engineering, SIMATS, Chennai, India. timeframe.

\section{LITERATURE SURVEY}

Incredible works have been made by scholarly system to automate retinal blood division increasingly exact retinal picture is being tried alongside derivation of different scientific parameters have been proposed however the exactness of the yield is low for instance Basic Simple Linear Iterative Clustering (SLIC) is the best in class calculation to section superpixels which doesn't require much computational power in SLIC the picture is isolated into pixels and the associated pixels are consolidated to frame superpixels and the picture surface is inspected [8]. Minimum spanning tree is utilized for bunch examination of the superpixels graphs and the superpixels are made SLIC delivers super pixels by gathering pixels reliant on their shading closeness and proximity in the image plane. In this method, the innate qualities of retinal pictures make the vein acknowledgement process troublesome.

\section{BLOCK DIAGRAM}

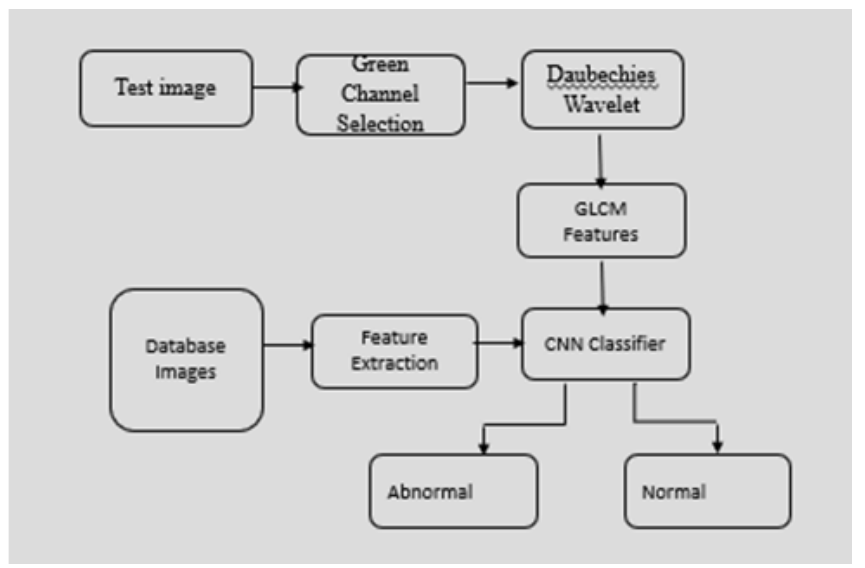

Fig. 1. Block diagram

\section{METHODOLOGY}

The Disease Classification by Retinal blood vessels is done by (i) Preprocessing of Retinal image, (ii) Feature Extraction, (iii)Morphological Filtering, (iv) Retinal vessel segmentation. The Pre-processing of the pupil image involves the green channel separation of the image. The Green image is extracted from the RGB images. And the extracted image is converted into Grayscale images for feature extraction and histogram equalization is done. 
The Morphological Filtering is done to the Grayscale images extracted from the test image. Retinal vessel segmentation involves dilated image sample and the eroded image is classified using spectral features. Daubechies wavelet transform is used for the Retinal vessel segmentation. Diabetic retinopathy is used for determining the disease in the blood vessel.
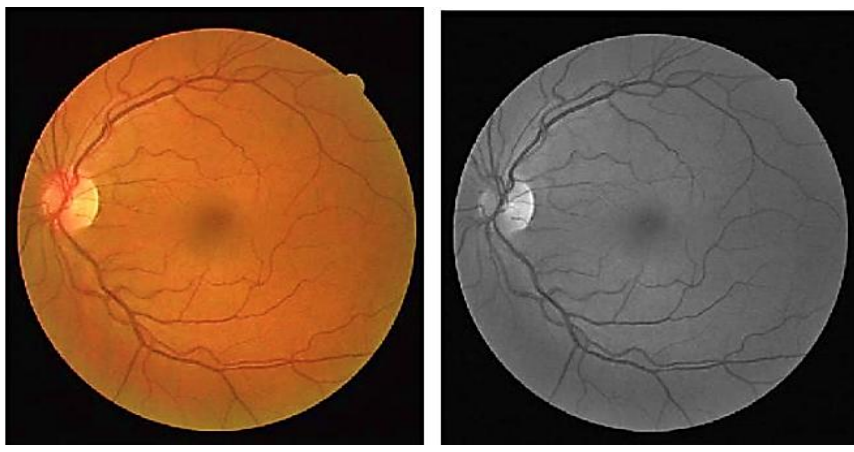

Fig. 2. (a) Normal retinal image and (b) Green component extracted image

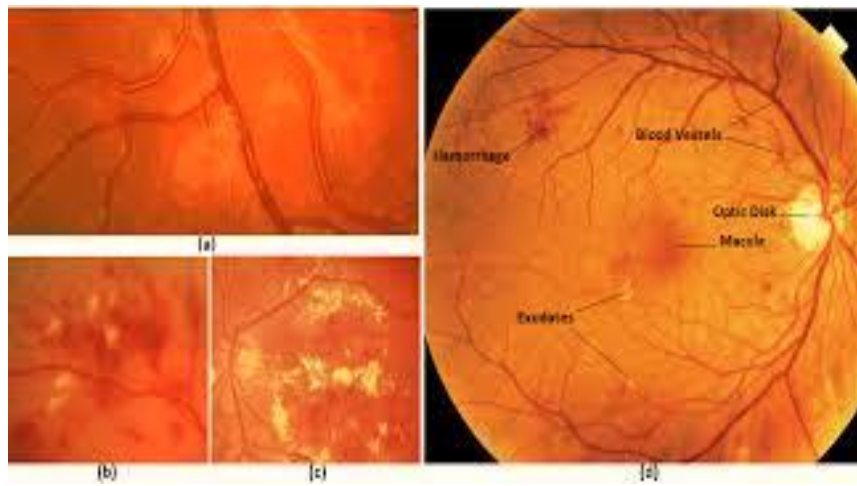

Fig. 3. Retinal blood vessel segmentation

\section{DIABETIC RETINOPATHY DIAGNOSIS}

The degenerative eye illness with the diabetic difficulties is most dangerous difficulty. The ophthalmologist made the decision by signs and manifestations of the diabetic retinopathy.

The signs of diabetic retinopathy are commonly not present amid the primary phase of the illness it can frequently go undiscovered until harm to vision has happened. This can be averted with yearly eye tests that incorporate a visual sharpness test, understudy enlargement, ophthalmoscopy, and tonometry tests. Since the essential driver of diabetic retinopathy is uncontrolled glucose levels, patients with sort I and II diabetes should verify that their vision is attempted reliably by an ophthalmologist.

Diabetic retinopathy happens when the minor veins, known as vessels, inside the retina are harmed. In patients with non-proliferative diabetic retinopathy (NPDR), the dividers of the vessel debilitate and create small scale aneurysms, or modest lumps projecting from the veins. In the long run, these small scale aneurysms start to spill blood and liquid into the retina, causing vision misfortune.
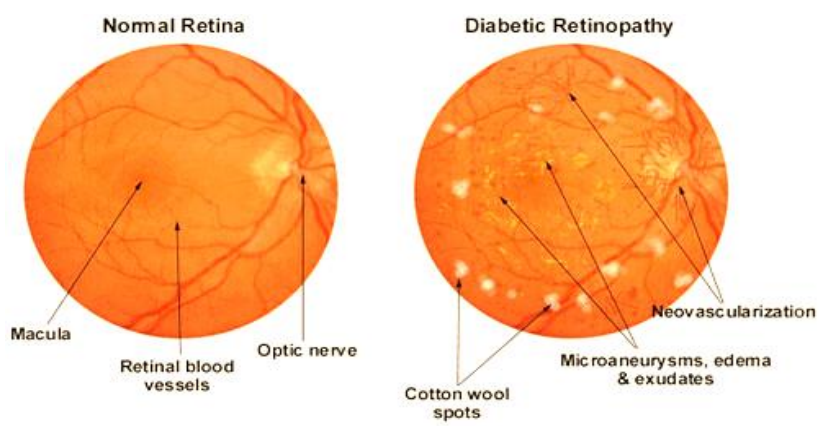

Fig. 4. Diabetic Retinopathy Diagnosis

\section{EXTRACTING THE GREEN IMAGE FROM THE RCB COMPONENT}

It is a kind of banner depiction gives the recurrent substance of flag at specific preview of time or spatial region. Stationary wavelet change isolates the picture into various subband pictures, it parts segment into various recurrent bundles called subbands. A high-repeat subband contains the edge data of information picture and LL subband contains the reasonable data about the picture, so we are going use Daubechies wavelet change in it to expand. Ingrid Daubechies, a champion among the most remarkable stars in the space of wavelet explore.

The names of the Daubechies family wavelets are made $\mathrm{dbN}$, where $\mathrm{N}$ is the interest, and $\mathrm{db}$ the "surname" of the wavelet. The $\mathrm{db} 1$ wavelet is relative to the Haar wavelet. the possible psi of the going with nine individuals from the family Db2, Db3, Db4, Db5, Db6, Db7, Db8, Db9, Db10.

\section{GLCM PROPERTIES}

In a factual surface examination, surface highlights processed from the measurable circulation watched mixes of powers at showed positions in regard to each other in the image. As demonstrated by the number of forces focuses (pixels) in each blend, experiences described into first-request, second-request and higher-request estimations.

The Gray Level Co-occurrence Matrix (GLCM) strategy is utilized for isolating second demand surface highlights. The system has used in different applications, third and higher request surfaces consider the associations among no less than three pixels. These are speculatively possible yet not routinely executed in light of tally time and understanding inconvenience.

\section{CONVOLUTIONAL NEURAL NETWORK CLASSIFIER (CNN)}

A convolutional neural system $(\mathrm{CNN})$ is a class of deep neural system, most usually connected to dissecting visual pictures. A convolutional neural system ( $\mathrm{CNN}$ 's) could be a composite of different rudimentary procedure units each that incorporates many weighted information sources and one yield, movement convolution of information signals with loads and improving the outcome with some style of nonlinearity. 
The units square measure sorted out in rectangular layers (grids).In this project, the CNN classifier gets the input database image which features are extracted by and the GLCM features are summed up and the Iteratively the images are classified by the CNN classifier to get the output whether normal or abnormal with respect to the diabetic spots on the retinal blood vessels of the eye.

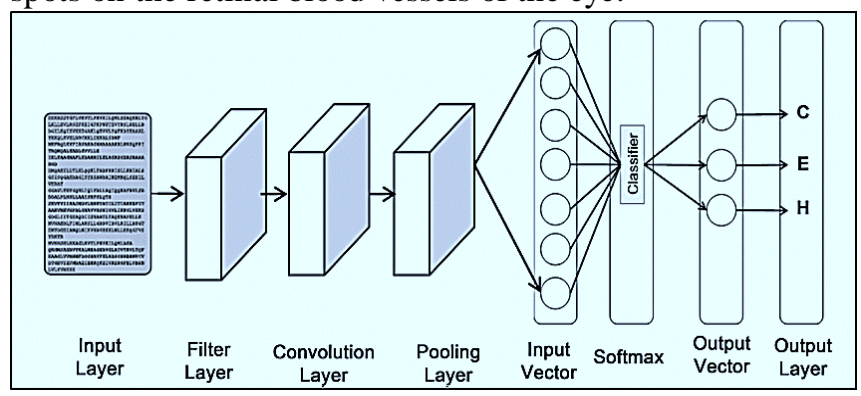

Fig. 5. bloodConvolutional Neural Network Classifier(CNN)

\section{WORKING}

This model is for identifying the diabetic retinopathy by examining the fundus picture utilizing Daubechies Wavelet and Neural Networks as Classifiers. The green direct extraction in the test picture (fundus picture) is segregated by the plane parcel system. The Green channel will have high affectability in the eye. The wavelet change gives the flag portrayal of the recurrence content in a flag at a specific moment of time or spatial area. The picture is gone through two integral channels and rises as two signs. Tragically, it might result in a multiplying of tests and subsequently to stay away from this, downsampling is presented. Which produces DWT coefficients. In this Daubechies wavelet is utilized. The Daubechies wavelet will bolster scaling and wavelet works that have extreme consistency for a given length of the help of the quadrature reflect channels. By this, the nerve edges of the picture can be followed. The extricating second request measurable surface highlights like Contrast and Homogeneity by utilizing the GLCM include. The expelled component data are given to the Neural framework classifier.The neural system classifier will contrast the test picture highlight information and the database pictures. In the event that the diabetic retinopathy distinguished. It will demonstrate the outcome anomalously and if not distinguished the outcome indicates ordinary.

\section{EXPERIMENTAL RESULT}

The Output has been acquired as normal or abnormal. It undergoes various processes like Edge Detection, Daubechies wavelets, Convolution Neural Network Transform and finally, it tells us what type of defect is present. The Above Results shows that the Eye is in either Normal state or Abnormal State. Both the states of Normal and Abnormal can be obtained in the Graphical User Interface. If the Result is acquired as abnormal the defect has been displayed in the Graphical User Interface.

\begin{tabular}{|l|l|l|l|l|l|}
\hline Test Image & Sensitivity & Specificity & Accuracy & RMSE & PSNR \\
\hline & 35.2761 & 70.1840 & 67.4041 & 0.1529 & 56.2862 \\
\hline & 39.1584 & 77.0914 & 74.8567 & 0.1357 & 56.8042 \\
\hline & & & & & \\
\hline
\end{tabular}

Fig. 6. Result and Discussion

$$
\begin{aligned}
& \text { Sensitivity }=\frac{\sum \mathrm{T}_{b}}{\sum \mathrm{T}_{b}+\sum \mathrm{F}_{\mathrm{nb}}} \\
& \text { Specificity }=\frac{\sum \mathrm{T}_{\mathrm{nb}}}{\sum \mathrm{T}_{\mathrm{nb}}+\sum \mathrm{F}_{\mathrm{b}}} \\
& \text { Accuracy }=\frac{\sum \mathrm{T}_{b}+\sum \mathrm{T}_{\mathrm{nb}}}{\sum \mathrm{T}_{\mathrm{nb}}+\sum \mathrm{F}_{\mathrm{b}}+\sum \mathrm{T}_{\mathrm{b}}+\sum \mathrm{F}_{\mathrm{nb}}}
\end{aligned}
$$

In this work we have found Sensitivity, Specificity, Accuracy, Root mean Square Error, power Signal to Noise Ratio for four images and some of the formulas of performance.

\section{CONCLUSION}

Through the Successful Utilization of this framework, it is conceivable to distinguish whether the Eye has been influenced by diabetic retinopathy or the eye has been in the ordinary territory of Sound State. Our proposed Framework is less support, Hazard free, Cost Effective. It will assist the general population by identifying that they were influenced by Diabetic retinopathy utilizing the adjustment in the measure of the retinal veins.

\section{REFERENCES}

1. B. Sheng, P. Li, S. Mo, H. Li, X. Hou, Q. Wu,J. Qin, R. Fang and D. D Feng, "Retinal vessel segmentation using minimum spanning superpixe tree detector", IEEE transactions on cybernetics, 49(7), May.2018, pp. 2707-2719.

2. S. Dua, N. Kandiraju and H. W. Thompson, "Design and implementation of a unique blood-vessel detection algorithm towards early diagnosis of diabetic retinopathy", In International Conference on Information Technology: Coding and Computing (ITCC'05)-Volume II (Vol. 1, Apr.2005, pp. 26-31).

3. A. M. Mendonc, and A. Campilho, "Segmentation of retinal blood vessels by combining the detection of centerlines and morphological reconstruction", IEEE transactions on medical imaging, 25(9), Agu.2006, pp.1200-1213.

4. A. Aquino, M. E. Gegúndez-Arias, and D. Marín, "Detecting the optic disc boundary in digital fundus images using morphological, edge detection, and feature extraction techniques", IEEE transactions on medical imaging, 29(11), Jun.2010, pp.1860-1869. 
5. M. Lalonde, M. Beaulieu, and L. Gagnon, " Fast and robust optic disc detection using pyramidal decomposition and Hausdorff-based template matching",IEEE transactions on medical imaging, 20(11), Nov.2001, pp.1193-1200.

6. T. R. Ganeshbabu, "Computer aided diagnosis of glaucoma detection using digital fundus image", International journal of advances in signal and image sciences, 1(1), Dec.2015, pp.1-11.

7. K. G. Kannan, T. R. Ganeshbabu, "Glaucoma image classification using discrete orthogonal stockwell transform", International journal of advances in signal and image sciences, 3(1), Jun.2017, pp. 6.

8. D. Maharaja, and D.S.M. Shaby, "Empirical Wavelet Transform and GLCM Features Based Glaucoma Classification from Fundus Image", International Journal of MC Square Scientific Research, Vol. 9, No. 1, 2017, pp. 79-85 